\title{
Pericardial Effusion Size
}

National Cancer Institute

\section{Source}

National Cancer Institute. Pericardial Effusion Size. NCI Thesaurus. Code C139047.

The size of a pericardial effusion as assessed at the point of greatest separation between the parietal and visceral pericardia. 